\title{
Isolation of Bacterial causes of Respiratory Infections in Calves in Smallholder farms in and around Gonder Town, Ethiopia
}

\section{Tefera Manaye}

Sede Woreda Veterinary University College of Fishery Science

Pawlos Wasihun Asnake ( $\nabla$ pwasihun@gmail.com )

Haramaya University https://orcid.org/0000-0002-3124-9082

Ashebr Abraha

Haramaya University

Tsegaw Fentie

university of Gondar

Research article

Keywords: Calves, Dairy farms, M. haemolytica, P. multocida, Respiratory disease

Posted Date: September 18th, 2020

DOI: https://doi.org/10.21203/rs.3.rs-69366/v1

License: (a) (i) This work is licensed under a Creative Commons Attribution 4.0 International License.

Read Full License 


\section{Abstract}

Background: Bovine respiratory disease (BRD) is considered as the major cause of severe respiratory tract infections in calves. Pasteurellosis is a multifactorial respiratory disease, which mainly affect calves within four weeks of weaning. A cross-sectional study was conducted from October 2017 to April 2018 in and around Gondar town, Amhara Regional State, North West of Ethiopia. The aim of the study was to isolate Mannheimia and Pasteurella species from calves up to six months old, and to assess the associated risk factors with the occurrence of respiratory disease. Sex, age ( $<16$ weeks and $>16$ weeks), body condition status (poor, medium, good), breed (local and cross breed), livelihood (mixed crop and urban), farming systems (semi intensive and intensive), herd size (small medium, and large), maternity pens (present or absent), and method of colostrum feedings (hand bucket and suckling) were the examined risk factors.

Results: A total of 84 nasopharyngeal swab samples were collected from calves with any signs of illness related to pasteurellosis. The overall isolation rate of the respiratory pathogens was $64 / 84(76.2 \%)(95 \%$ $\mathrm{Cl}=65.7-84.8$ ), with $46.4 \%$ of Mannheimia haemolytica and $28.8 \%$ Pasteurella multocida isolates. The distribution of pathogens was statistically higher $(P<0.001)$ in calves with respiratory problems $(93.6 \%$; $95 \% \mathrm{Cl}=82.5-98.7)$ compared to those with no symptoms of respiratory illness $(54.1 \% ; 95 \% \mathrm{Cl}=36.9$ 70.5). Among the examined risk factors age, sex, breed, farming system were found to be potential risk factors and significantly associated with Pasteurella infection of calves $(\mathrm{p}<0.05)$. The higher isolation rate of Mannheimia haemolytica indicated that it is the major cause of respiratory disease in the study area.

Conclusion: The present finding revealed that pasteurellosis is one of the major diseases of calves in the study area in which $M$. haemolytica and $P$. multocida were found to be commonly involved in respiratory infections. Improved farm management including timely feeding of colostrum, appropriate hygiene of the calf house and training of farmers is recommended to prevent and control of respiratory diseases in the study area.

\section{Background}

Bovine respiratory disease (BRD) is considered as the major cause of severe respiratory tract infections in calves [1]. It causes great economic losses such as reduced average daily gain, feed efficiency, and overall performance of beef calves and finally calves morbidity and mortality [2,3]. Several infectious agents are commonly isolated from the respiratory tracts of clinically sick and healthy animals [4]. Many factors can weaken the host's immune system and/or damage the lining of the respiratory tract to such an extent that these pathogens are able to progress deeper into the respiratory tract and cause disease [5-6].

Calf pneumonia refers to infectious respiratory disease in calves. It is primarily a problem in calves less than 6 months old with peak occurrence from 2-10 weeks, but it may be seen in calves up to 1 year of 
age [7]. Pasteurellosis is a multifactorial respiratory disease [6, 8], which mainly affect calves within four weeks of weaning. Infected calves manifest the clinical signs when they are sorted and sold to different farms. The bacteria that causes pasteurellosis are part of the normal microbiota in the upper respiratory tract [9], making the disease difficult to prevent. mannheimia haemolytica and pasteurella multocida are most commonly associated with pneumonia in cattle calves $[10,11]$. These pathogens can easily spread between animals, especially when calves are crowded (as in shipment) or closely confined (as in a dairy calf nursery) [12].

In practice, deep nasopharyngeal swabs (DNS) [13], transtracheal aspiration (TTA) [14], and broncho alveolar lavage (BAL) [15] have been used for sampling the respiratory tract. Deep nasopharyngeal swab is the easiest, fastest, and cheapest technique and therefore most suitable for sampling large numbers of animals [16].

In Ethiopia, calf pneumonia has been reported as the second most disease syndrome associated with calf morbidity and mortality next to calf diarrhea $[17,18]$. Even though bovine pneumonic pasteurellosis is one of the most economically important infectious diseases in Ethiopia [19], there is limited data on the status of the disease and potential risk factors. This is particularly important in the study area, because dairy farms are an integral part of the economy. Hence, the study was conducted to identify the main bacterial respiratory pathogens and to assess major risk factors in bovine calves managed under dairy farms of Gondar town.

\section{Methods}

\section{Study area}

Sample collection was held in and around Gondar town, which is the capital of central Gondar administration zone, Amhara regional state. The area is located in the north west of Ethiopia at $740 \mathrm{~km}$ from Addis Ababa, capital city of the country. It is situated between $12^{\circ} 36^{\prime} \mathrm{N}$ and $33^{\circ} 28^{\prime} \mathrm{E}$ at an altitude of about $2300 \mathrm{~m}$ above mean sea level with an average temperature of $20^{\circ} \mathrm{C}$ and an average annual rain fall of $1800 \mathrm{~mm}$.

\section{Study Population}

The study population constitutes bovine calves up to 6 months of age that shows any respiratory sign or having general illness (suspected to have respiratory pathogens) found in dairy farms located at urban as well as peri-urban, which is characterized by mixed crop livestock production system. Study animals are selected regardless of breed, sex, body condition, herd size, and weaning status.

\section{Study Design and Animal Selection}

A cross-sectional study was employed to isolate bacterial pathogens causing bovine respiratory disease of calves and assess its major risk factors from November 2017 to April 2018. Purposive type of 
sampling was applied based on the availability of calves and willingness of farmers to take the nasopharyngeal swabs for bacteriological culture of Pasteurella species. Thus, a total of 84 calves were included in the study.

\section{Sampling Procedures}

Animals were restrained by an assistant and then the external part of the nose was disinfected with $70 \%$ alcohol. After the alcohol has evaporated, a sterile cotton-tipped swab was inserted in to the nostril and rotated against the wall of the nasal cavity as described by [20]. The swab was then placed in a labeled sterile test tube containing $3 \mathrm{ml}$ of tryptose soya broth. Samples were then kept in a box containing ice for transport to University of Gondar veterinary microbiology laboratory.

\section{Isolation and identification of Pasteurella species}

The isolation and identification was performed as described by [21]. Briefly, the specimen in tryptose soya broth was incubated for 24 hours at $37^{\circ} \mathrm{C}$ and a loop full of the broth culture was streak on petri-dish of blood agar base supplemented with $5 \%$ sheep blood then incubated aerobically at $37^{\circ} \mathrm{C}$ for 24 hours. Then colonies showing typical gram's reaction and cellular morphology were further sub-culture on both blood and MacConkey agar plates. The general appearance of colonies, presence and nature of hemolysis, and the ability to ferment lactose was recorded. The suspected pure colonies from both blood and MacConkey agars were transferred onto nutrient agar slants for further identification by biochemical tests. M. haemolytica was characterized as able to produce a narrow zone of hemolysis on blood agar, able to grow on MacConkey agar, but unable to produce indole, whereas $P$. multocida was characterized as unable to produce hemolysis on blood agar, unable to grow on MacConkey agar, and able to produce indole as described by [22].

\section{Data management and Statistical Analysis}

Data was stored in Microsoft excel sheet for handling. After checking for its correctness, it was transferred to SPSS version 20.0 for analysis. Descriptive analysis, such as frequency and percentage were used to describe the proportion of calves affected by bacteria. Chi-square and regression analysis were employed to establish the association between cultured bacteria result and risk factors. Before regression analysis, the data was cheeked for fulfillments of assumptions, such as correlation of each variables (not more than 0.7), correlation of independent variables with dependent variable (minimum of $0.3)$, and multi-collinearity tests (VIF ( $>10)$ and Tolerance $(<0.1)$ ). Thus, Age, sex, and breed were used in the final model. The OR was computed at $95 \% \mathrm{Cl}$ and in all cases the difference between parameters were tested for significance at probability level of less than $0.05(P \leq 0.05)$.

\section{Results}

\section{Overall Prevalence of Isolates}

Physical examination indicated that 47 (55.9\%) and 37 (44.1\%) animals were with signs of respiratory illness and with no symptoms of respiratory illness, respectively. Out of 84 calves examined, $64(76.2 \%)$ 
were found to harbor Pasteurella species and the prevalence was significantly higher $(\mathrm{p}<0.001)$ in animals with symptoms of respiratory illness (Table 1). Meanwhile, the proportion of isolated Mannheimia haemolytica (46.4\%) was higher than Pasteurella multocida (28.8\%) (Table 2).

Table 1

Summary of total isolate with corresponding health status

\begin{tabular}{|llll|}
\hline Health status & No. of positive samples $(\%)$ & $95 \% \mathrm{Cl}$ & \multirow{2}{*}{ X2 value (p-value) } \\
\hline No-respiratory problems $(\mathrm{n}=37)$ & $20(54.1)$ & $36.9-70.5$ & \multirow{2}{*}{$17.86(0.000)$} \\
\cline { 1 - 3 } With respiratory problems $(\mathrm{n}=47)$ & $44(93.6)$ & $82.5-98.7$ & \\
\cline { 1 - 4 } Total $(\mathrm{n}=84)$ & $64(76.2)$ & $65.7-84.8$ & \\
\hline
\end{tabular}

Table 2

Distribution of Pasteurella species isolated from nasopharyngeal swabs of calves $(n=84)$

\begin{tabular}{|lll|}
\hline Species of Pasteurella & Number of positive samples & $\%$ of isolates \\
\hline M. haemolytica & 39 & $46.4 \%$ \\
\hline P. multocida & 257 & $28.8 \%$ \\
\hline Over all & 64 & $76.2 \%$ \\
\hline
\end{tabular}

The prevalence of pasteurellosis in relation to risk factors revealed a statistically significant association with age $(p=0.05)$, breed $(p=0.05)$, and livelihood $(p=0.03)$. The occurrence was higher in male $(84.1 \%)$ than female calves (67.5\%); in young (82.5\%) than older calves (63\%); in cross breed (82.5\%) compared to local bred (63\%); and urban (82.8\%) than peri-urban (mixed crop productions) areas (61.5\%) (Table 3). Based on logistic regression analysis, the odds of being affected by pasteurellosis was significantly higher in calves aged $<16$ weeks $(O R=3.1 ; 95 \% \mathrm{Cl}=1.0-9.6 ; \mathrm{P}=0.04)$ than the older category and crossbred than local one $(\mathrm{OR}=3.6 ; 95 \% \mathrm{Cl}=1.1-11.5 ; \mathrm{P}=0.03)($ Table 4$)$. 
Table 3

Pasteurellosis in calves and associated risk factors

\begin{tabular}{|c|c|c|c|c|c|}
\hline \multirow[t]{2}{*}{ Variables } & \multirow{2}{*}{$\begin{array}{l}\text { No. of Animals } \\
\text { examined }\end{array}$} & \multirow{2}{*}{$\begin{array}{l}\text { No of Animals with } \\
\text { Pathogens (\%) }\end{array}$} & \multirow[t]{2}{*}{$\mathrm{Cl}(95 \%)$} & \multicolumn{2}{|c|}{$\mathrm{X} 2$ analysis } \\
\hline & & & & $\begin{array}{l}x^{2} \\
\text { value }\end{array}$ & $\begin{array}{l}\mathrm{p}- \\
\text { value }\end{array}$ \\
\hline \multicolumn{6}{|l|}{ Sex } \\
\hline Female & 40 & $27(67.5)$ & $\begin{array}{l}50.9- \\
81.4\end{array}$ & \multirow[t]{2}{*}{3.2} & \multirow[t]{2}{*}{0.075} \\
\hline Male & 44 & $37(84.1)$ & $\begin{array}{l}69.9- \\
93.4\end{array}$ & & \\
\hline \multicolumn{6}{|l|}{ Age } \\
\hline$<16$ weeks & 57 & $47(82.5)$ & $\begin{array}{l}70.1- \\
91.3\end{array}$ & \multirow[t]{2}{*}{3.8} & \multirow[t]{2}{*}{0.05} \\
\hline$\geq 16$ weeks & 27 & $17(63.0)$ & $\begin{array}{l}42.4- \\
80.6\end{array}$ & & \\
\hline \multicolumn{6}{|l|}{ BCS } \\
\hline Poor & 13 & $10(76.9)$ & $\begin{array}{l}46.2- \\
94.9\end{array}$ & \multirow[t]{3}{*}{0.005} & \multirow[t]{3}{*}{0.99} \\
\hline Medium & 50 & $38(76.0)$ & $\begin{array}{l}61.8- \\
86.9\end{array}$ & & \\
\hline Good & 21 & $16(76.2)$ & $\begin{array}{l}52.8- \\
91.8\end{array}$ & & \\
\hline \multicolumn{6}{|l|}{ Breed } \\
\hline Local & 27 & $17(63.0)$ & $\begin{array}{l}42.7- \\
80.6\end{array}$ & \multirow[t]{2}{*}{3.8} & \multirow[t]{2}{*}{0.05} \\
\hline Cross-breed & 57 & $47(82.5)$ & $\begin{array}{l}70.1- \\
91.3\end{array}$ & & \\
\hline \multicolumn{6}{|l|}{ Livelihood } \\
\hline Mixed crop & 26 & $16(61.5)$ & $\begin{array}{l}40.6- \\
79.8\end{array}$ & \multirow[t]{2}{*}{4.46} & \multirow[t]{2}{*}{0.035} \\
\hline Urban & 58 & $48(82.8)$ & $\begin{array}{l}70.6- \\
91.4\end{array}$ & & \\
\hline \multicolumn{6}{|l|}{ Farming system } \\
\hline $\begin{array}{l}\text { Semi- } \\
\text { intensive }\end{array}$ & 34 & $24(70.6)$ & $\begin{array}{l}52.5- \\
84.9\end{array}$ & \multirow[t]{2}{*}{0.98} & \multirow[t]{2}{*}{0.32} \\
\hline Intensive & 50 & $40(80.0)$ & $\begin{array}{l}66.3- \\
89.9\end{array}$ & & \\
\hline
\end{tabular}




\begin{tabular}{|c|c|c|c|c|c|}
\hline \multirow[t]{2}{*}{ Variables } & \multirow{2}{*}{$\begin{array}{l}\text { No. of Animals } \\
\text { examined }\end{array}$} & \multirow{2}{*}{$\begin{array}{l}\text { No of Animals with } \\
\text { Pathogens (\%) }\end{array}$} & \multirow[t]{2}{*}{$\mathrm{Cl}(95 \%)$} & \multicolumn{2}{|c|}{$\chi 2$ analysis } \\
\hline & & & & $\begin{array}{l}\chi 2 \\
\text { value }\end{array}$ & $\begin{array}{l}\mathrm{p}- \\
\text { value }\end{array}$ \\
\hline \multicolumn{6}{|l|}{ Herd size } \\
\hline Small & 12 & $9(75.0)$ & $\begin{array}{l}42.8- \\
94.5\end{array}$ & \multirow[t]{2}{*}{0.46} & \multirow[t]{2}{*}{0.79} \\
\hline Medium & 59 & $46(78.0)$ & $\begin{array}{l}65.3- \\
87.7\end{array}$ & & \\
\hline Large & 13 & $9(69.2)$ & $\begin{array}{l}38.6- \\
90.9\end{array}$ & & \\
\hline \multicolumn{6}{|l|}{ Maternity pen } \\
\hline Present & 27 & $18(66.7)$ & $\begin{array}{l}46.0- \\
83.5\end{array}$ & \multirow[t]{2}{*}{1.9} & \multirow[t]{2}{*}{0.16} \\
\hline Absent & 57 & $46(80.7)$ & $\begin{array}{l}69.6- \\
91.1\end{array}$ & & \\
\hline \multicolumn{6}{|l|}{ MCF } \\
\hline Suckling & 66 & $50(75.8)$ & $\begin{array}{l}63.6- \\
85.5\end{array}$ & \multirow[t]{2}{*}{0.03} & \multirow[t]{2}{*}{0.86} \\
\hline Hand/bucket & 18 & $14(77.8)$ & $\begin{array}{l}52.4- \\
93.6\end{array}$ & & \\
\hline $\mathrm{Cl}=$ Confidence in & al; BCS = Body & ion status; $M C F=M \epsilon$ & lostrum $t$ & ding. & \\
\hline
\end{tabular}


Table 4

Regression analysis of pasteurellosis with associated risk factors

\begin{tabular}{|c|c|c|c|c|}
\hline \multirow[t]{2}{*}{ Variables } & \multirow{2}{*}{$\begin{array}{l}\text { No. of Animals } \\
\text { examined }\end{array}$} & \multirow{2}{*}{$\begin{array}{l}\text { No of Animals with } \\
\text { pathogens (\%) }\end{array}$} & \multicolumn{2}{|c|}{ Multivariable LG analysis } \\
\hline & & & $\begin{array}{l}\text { Odds ratio }(95 \% \\
\mathrm{Cl})\end{array}$ & $\begin{array}{l}\mathrm{p} \text { - } \\
\text { value }\end{array}$ \\
\hline \multicolumn{5}{|l|}{ Sex } \\
\hline Female & 40 & $27(67.5)$ & * & \\
\hline Male & 44 & $37(84.1)$ & $1.64(0.52-5.2)$ & 0.4 \\
\hline \multicolumn{5}{|l|}{ Age } \\
\hline $\begin{array}{l}\geq 16 \\
\text { weeks }\end{array}$ & 27 & $17(63.0)$ & * & \\
\hline $\begin{array}{l}<16 \\
\text { weeks }\end{array}$ & 57 & $47(82.5)$ & $3.1(1.0-9.6)$ & 0.04 \\
\hline \multicolumn{5}{|l|}{ Breed } \\
\hline $\begin{array}{l}\text { Cross- } \\
\text { bred }\end{array}$ & 27 & $17(63.0)$ & * & \\
\hline Local & 57 & $47(82.5)$ & $3.6(1.1-11.5)$ & 0.03 \\
\hline
\end{tabular}

\section{Prevalence of Pasteurella species and Risk Factors}

The result showed that $M$. haemolytica and $P$. multocida varied in proportion with different factors examined. Thus, sex and health status were significant associated $(P<0.05)$ with the occurrence of $M$. haemolytica, in that it was higher in male (56.8\%) than female calves (35.0\%) and animals with signs of respiratory illness (57.4\%) than healthy one (32.4\%). Moreover, the prevalence was relatively higher in calves of $<16$ weeks old $(50.9 \%)$ than older calves (37.0\%) (Table 5). With regard to P. multocida, the occurrence was higher in cross bred (33.3\%) than local $(22.2 \%)$ and calves with signs respiratory illness (36.2\%) than healthy one (21.6\%) (Table 6). 
Table 5

M. haemolytica infection in calves and associated risk factors

\begin{tabular}{|c|c|c|c|c|c|}
\hline \multirow[t]{2}{*}{ Variables } & \multirow{2}{*}{$\begin{array}{l}\text { No. of Animals } \\
\text { examined }\end{array}$} & \multirow{2}{*}{$\begin{array}{l}\text { No of Animals with } M \text {. } \\
\text { haemolytica (\%) }\end{array}$} & \multirow{2}{*}{$\begin{array}{l}\mathrm{Cl} \\
(95 \%)\end{array}$} & \multicolumn{2}{|c|}{$\mathrm{X} 2$ analysis } \\
\hline & & & & $\begin{array}{l}\chi^{2} \\
\text { value }\end{array}$ & $\begin{array}{l}\mathrm{p}- \\
\text { value }\end{array}$ \\
\hline \multicolumn{6}{|l|}{ Sex } \\
\hline Female & 40 & $14(35.0)$ & $\begin{array}{l}20.6- \\
51.7\end{array}$ & \multirow[t]{2}{*}{4.01} & \multirow[t]{2}{*}{0.045} \\
\hline Male & 44 & $25(56.8)$ & $\begin{array}{l}41.0- \\
71.7\end{array}$ & & \\
\hline \multicolumn{6}{|l|}{ Age } \\
\hline$<16$ weeks & 57 & $29(50.9)$ & $\begin{array}{l}37.3- \\
64.4\end{array}$ & \multirow[t]{2}{*}{1.4} & \multirow[t]{2}{*}{0.23} \\
\hline$\geq 16$ weeks & 27 & $10(37.0)$ & $\begin{array}{l}19.4- \\
57.6\end{array}$ & & \\
\hline \multicolumn{6}{|l|}{ BCS } \\
\hline Poor & 13 & $5(38.5)$ & $\begin{array}{l}13.9- \\
68.4\end{array}$ & \multirow[t]{3}{*}{0.64} & \multirow[t]{3}{*}{0.73} \\
\hline Medium & 50 & $23(46.0)$ & $\begin{array}{l}31.8- \\
60.7\end{array}$ & & \\
\hline Good & 21 & $11(52.4)$ & $\begin{array}{l}29.9- \\
74.3\end{array}$ & & \\
\hline \multicolumn{6}{|l|}{ Breed } \\
\hline Local & 27 & $11(40.7)$ & $\begin{array}{l}22.4- \\
61.2\end{array}$ & \multirow[t]{2}{*}{0.52} & \multirow[t]{2}{*}{0.47} \\
\hline Cross-bred & 57 & $28(49.1)$ & $\begin{array}{l}35.6- \\
62.7\end{array}$ & & \\
\hline \multicolumn{6}{|l|}{$\begin{array}{l}\text { Respiratory } \\
\text { illness }\end{array}$} \\
\hline Absent & 37 & $12(32.4)$ & $\begin{array}{l}18.0- \\
49.8\end{array}$ & \multirow[t]{2}{*}{5.2} & \multirow[t]{2}{*}{0.02} \\
\hline Present & 47 & $27(57.4)$ & $\begin{array}{l}42.2- \\
71.7\end{array}$ & & \\
\hline \multicolumn{6}{|l|}{ Livelihood } \\
\hline Mixed crop & 26 & $11(42.3)$ & $\begin{array}{l}23.4- \\
63.1\end{array}$ & 0.25 & 0.61 \\
\hline
\end{tabular}




\begin{tabular}{|c|c|c|c|c|c|}
\hline \multirow[t]{2}{*}{ Variables } & \multirow{2}{*}{$\begin{array}{l}\text { No. of Animals } \\
\text { examined }\end{array}$} & \multirow{2}{*}{$\begin{array}{l}\text { No of Animals with } M \text {. } \\
\text { haemolytica (\%) }\end{array}$} & \multirow{2}{*}{$\begin{array}{l}\mathrm{Cl} \\
(95 \%)\end{array}$} & \multicolumn{2}{|c|}{$\mathrm{X} 2$ analysis } \\
\hline & & & & $\begin{array}{l}x 2 \\
\text { value }\end{array}$ & $\begin{array}{l}\mathrm{p}- \\
\text { value }\end{array}$ \\
\hline Urban & 58 & $28(48.3)$ & $\begin{array}{l}34.9- \\
61.8\end{array}$ & & \\
\hline \multicolumn{6}{|l|}{ Farming system } \\
\hline $\begin{array}{l}\text { Semi- } \\
\text { intensive }\end{array}$ & 34 & $17(50.0)$ & $\begin{array}{l}32.4- \\
67.6\end{array}$ & \multirow[t]{2}{*}{0.29} & \multirow[t]{2}{*}{0.58} \\
\hline Intensive & 50 & $22(44.0)$ & $\begin{array}{l}29.9- \\
58.7\end{array}$ & & \\
\hline \multicolumn{6}{|l|}{ Herd size } \\
\hline Small & 12 & $5(41.7)$ & $\begin{array}{l}15.2- \\
72.3\end{array}$ & \multirow[t]{3}{*}{0.13} & \multirow[t]{3}{*}{0.93} \\
\hline Medium & 59 & $28(47.5)$ & $\begin{array}{l}34.3- \\
60.9\end{array}$ & & \\
\hline Large & 13 & $6(46.2)$ & $\begin{array}{l}19.2- \\
74.9\end{array}$ & & \\
\hline \multicolumn{6}{|l|}{ Maternity pen } \\
\hline Present & 27 & $12(44.4)$ & $\begin{array}{l}25.5- \\
64.7\end{array}$ & \multirow[t]{2}{*}{0.06} & \multirow[t]{2}{*}{0.80} \\
\hline Absent & 57 & $27(47.4)$ & $\begin{array}{l}33.9- \\
61.0\end{array}$ & & \\
\hline \multicolumn{6}{|l|}{ MCF } \\
\hline Suckling & 66 & $29(43.9)$ & $\begin{array}{l}31.7- \\
56.7\end{array}$ & \multirow[t]{2}{*}{0.76} & \multirow[t]{2}{*}{0.38} \\
\hline Hand/bucket & 18 & $10(55.6)$ & $\begin{array}{l}30.8^{-} \\
78.5\end{array}$ & & \\
\hline
\end{tabular}


Table 6

P. multocida infection in calves and associated risk factors

\begin{tabular}{|c|c|c|c|c|c|}
\hline \multirow[t]{2}{*}{ Variables } & \multirow{2}{*}{$\begin{array}{l}\text { No. of Animals } \\
\text { examined }\end{array}$} & \multirow{2}{*}{$\begin{array}{l}\text { No of Animals with } P \\
\text { multocida (\%) }\end{array}$} & \multirow{2}{*}{$\begin{array}{l}\mathrm{Cl} \\
(95 \%)\end{array}$} & \multicolumn{2}{|c|}{$\mathrm{X} 2$ analysis } \\
\hline & & & & $\begin{array}{l}\chi^{2} \\
\text { value }\end{array}$ & $\begin{array}{l}\mathrm{p}- \\
\text { value }\end{array}$ \\
\hline \multicolumn{6}{|l|}{ Sex } \\
\hline Female & 40 & $13(32.5)$ & $\begin{array}{l}18.6- \\
49.1\end{array}$ & \multirow[t]{2}{*}{0.27} & \multirow[t]{2}{*}{0.61} \\
\hline Male & 44 & $12(27.3)$ & $\begin{array}{l}14.9- \\
42.8\end{array}$ & & \\
\hline \multicolumn{6}{|l|}{ Age } \\
\hline$<16$ weeks & 57 & 18 (31.6) & $\begin{array}{l}19.9- \\
45.2\end{array}$ & \multirow[t]{2}{*}{0.28} & \multirow[t]{2}{*}{0.59} \\
\hline$\geq 16$ weeks & 27 & 7 (25.9) & $\begin{array}{l}11.1- \\
46.3\end{array}$ & & \\
\hline \multicolumn{6}{|l|}{ BCS } \\
\hline Poor & 13 & 5 (38.5) & $\begin{array}{l}13.9- \\
68.4\end{array}$ & \multirow[t]{3}{*}{0.83} & \multirow[t]{3}{*}{0.66} \\
\hline Medium & 50 & $15(30.0)$ & $\begin{array}{l}17.9- \\
44.6\end{array}$ & & \\
\hline Good & 21 & $5(23.8)$ & $\begin{array}{l}8.2- \\
47.2\end{array}$ & & \\
\hline \multicolumn{6}{|l|}{ Breed } \\
\hline Local & 27 & $6(22.2)$ & $\begin{array}{l}08.6- \\
42.3\end{array}$ & \multirow[t]{2}{*}{1.08} & \multirow[t]{2}{*}{0.29} \\
\hline Cross-bred & 57 & 19 (33.3) & $\begin{array}{l}21.4- \\
47.1\end{array}$ & & \\
\hline \multicolumn{6}{|l|}{$\begin{array}{l}\text { Respiratory } \\
\text { illness }\end{array}$} \\
\hline Absent & 37 & 8 (21.6) & $\begin{array}{l}09.8- \\
38.2\end{array}$ & \multirow[t]{2}{*}{2.09} & \multirow[t]{2}{*}{0.15} \\
\hline Present & 47 & $17(36.2)$ & $\begin{array}{l}22.7- \\
51.5\end{array}$ & & \\
\hline \multicolumn{6}{|l|}{ Livelihood } \\
\hline Mixed crop & 26 & 5 (19.2) & $\begin{array}{l}6.6- \\
39.4\end{array}$ & 1.99 & 0.16 \\
\hline
\end{tabular}




\begin{tabular}{|c|c|c|c|c|c|}
\hline \multirow[t]{2}{*}{ Variables } & \multirow{2}{*}{$\begin{array}{l}\text { No. of Animals } \\
\text { examined }\end{array}$} & \multirow{2}{*}{$\begin{array}{l}\text { No of Animals with } P . \\
\text { multocida (\%) }\end{array}$} & \multirow{2}{*}{$\begin{array}{l}\mathrm{Cl} \\
(95 \%)\end{array}$} & \multicolumn{2}{|c|}{$\chi 2$ analysis } \\
\hline & & & & $\begin{array}{l}\chi^{2} \\
\text { value }\end{array}$ & $\begin{array}{l}\mathrm{p}- \\
\text { value }\end{array}$ \\
\hline Urban & 58 & $20(34.5)$ & $\begin{array}{l}22.5- \\
48.1\end{array}$ & & \\
\hline \multicolumn{6}{|l|}{ Farming system } \\
\hline $\begin{array}{l}\text { Semi- } \\
\text { intensive }\end{array}$ & 34 & 7 (20.6) & $\begin{array}{l}08.7- \\
37.9\end{array}$ & \multirow[t]{2}{*}{2.3} & \multirow[t]{2}{*}{0.14} \\
\hline Intensive & 50 & $18(36.0)$ & $\begin{array}{l}22.9- \\
50.8\end{array}$ & & \\
\hline \multicolumn{6}{|l|}{ Herd size } \\
\hline Small & 12 & $4(33.3)$ & $\begin{array}{l}9.9- \\
65.1\end{array}$ & \multirow[t]{3}{*}{0.37} & \multirow[t]{3}{*}{0.83} \\
\hline Medium & 59 & $18(30.5)$ & $\begin{array}{l}19.2- \\
43.9\end{array}$ & & \\
\hline Large & 13 & $3(23.1)$ & $\begin{array}{l}05.0 \\
-53.8\end{array}$ & & \\
\hline \multicolumn{6}{|l|}{ Maternity pen } \\
\hline Present & 27 & $6(22.2)$ & $\begin{array}{l}8.6- \\
42.3\end{array}$ & \multirow[t]{2}{*}{1.08} & \multirow[t]{2}{*}{0.29} \\
\hline Absent & 57 & 19 (33.3) & $\begin{array}{l}21.4- \\
47.1\end{array}$ & & \\
\hline \multicolumn{6}{|l|}{ MCF } \\
\hline Suckling & 66 & $21(31.8)$ & $\begin{array}{l}20.9- \\
44.4\end{array}$ & \multirow[t]{2}{*}{0.62} & \multirow[t]{2}{*}{0.43} \\
\hline Hand/bucket & 18 & $4(22.2)$ & $\begin{array}{l}6.4- \\
47.6\end{array}$ & & \\
\hline
\end{tabular}

\section{Cultural and Biochemical Characteristics of Isolated Pasteurella Species}

Mannheimia haemolytica were able to grow as small red colony on MacConkey agar and show $\beta$ hemolysis on blood agar, while Pasteurella multocida were unable to grow on MacConkey agar and nonhemolytic (Table 7), but had mucoid colony and gram negative on gram staining. Different biochemical tests performed to identify the isolated pathogen and all the isolates were positive for oxidase, catalase, nitrate and phosphate and able to ferment sucrose, glucose and mannose. P. multocida isolates were positive for indole test but unable to ferment lactose and maltose (Table 7). 
Table 7

Results of biochemical characteristics of bacterial species isolated from calves

\begin{tabular}{|lll|}
\hline Tests & Species & \\
\cline { 2 - 3 } & P. multocida & M. haemolytica \\
\hline Growth on MacConkey agar & - & + \\
\hline Hemolysis on sheep blood agar & - & + \\
\hline Indole production & + & - \\
\hline Oxidase & + & + \\
\hline Catalase & + & + \\
\hline Nitrate & + & + \\
\hline Phosphatase & + & + \\
\hline Urease & - & - \\
\hline Acid from: & & + \\
\hline Lactose & - & + \\
\hline Maltose & - & + \\
\hline Sucrose & + & + \\
\hline Mannose & + & + \\
\hline
\end{tabular}

\section{Discussion}

In the present study the overall isolation rate of pasteurellosis was found to be $76.2 \%(95 \% \mathrm{Cl}=65.7-$ 84.8). The current finding in the prevalence of pasteurellosis is relatively higher than reports of previous studies in the country. Thus, [23], [24], [25] [26], and [27], who 50.2, 40.8, 39.2, 13, and 8.7\%, respectively. This might be due to the difference in sampling that this study was conducted on cases and suspected of pneumonia, study area, time of sampling and farm management [23].

Analysis on sex related susceptibility showed that pasteurellosis was higher in male (84.1\%) than female $(67.5 \%)$ calves, in that the odd of being positive was 1.64 times higher in the former $(\mathrm{OR}=1.64 ; 95 \% \mathrm{Cl}=$ $0.52-5.2)$. The possible explanation for sex related susceptibility is that less colostral immunoglobulin absorbed in male than female during neonatal life which leads to disease in male calves [28]. It is also worth mentioning that male calves are not as valuable to the dairy operation as females and therefore may not receive an attention the heifers do have, possibly accounting for the higher infection in males $[29,30]$. Meanwhile, the prevalence was significantly higher in younger calves $(<16$ weeks) $(82.5 \%)$ than older one ( $\geq 16$ weeks) $(63.0 \%)$ with younger calves have 3.1 times the chances of being affected than 
older $(\mathrm{OR}=3.1 ; 95 \% \mathrm{Cl}=1.0-9.6 ; \mathrm{p}=0.04)$. The possible explanation for the age-related susceptibility might be due to failure of passive immunity in hand feeding practices of young calves being able to predispose for bacterial infection and other predisposing etiological agents [21]. For instance, calves less than one month of age lack sufficient postruminal digestive enzymes to break down most sugars and are limited in their ability to utilize starch, maltose, sucrose, or dextran [31]. Partial or complete failure of passive transfer of maternal antibodies is an important host factor related to development of pneumonia in young calves $[7,32]$. There was significant association $(p<0.05)$ between pasteurellosis and breed of calves, with higher prevalence in cross breed $(82.5 \%)$ than local $(63 \%)$ calves. This deference might be linked to variation in environment adaptability. Therefore, local breed has high disease resistant capability. Similarly, the difference in the isolation rate of the two breeds might be due to the difference in feed access of the calves. Local breeds feed relatively less in amount and quality than cross breed calves which consume much amount and quality feed. So local breeds being less exposed to infection and therefore, have lower isolation rate than cross breed [33].

This study compares the level of isolation rate between animals kept under intensive and semi intensive management systems. The infection rate was higher in calves kept under intensive system (80\%) compared to semi intensive one (70.6\%). An intensive management system is mainly associated with confinements and predisposing calves are frequently contact to accumulations of urine and other wastes. This situation is likely to favors the spread of Pasteurella species among animals [29]. Similar observation was reported previously by [34;35]. Moreover, the occurrence of pasteurellosis varied significantly $(p=0.035)$ among livelihood, in that it was higher in urban $(82.8 \%)$ than mixed crop $(61.5 \%)$ production system.

The overall proportion of Mannheimia haemolytica and Pasteurella multocida was $46.4 \%$ and $28.8 \%$, respectively, indicating $M$. haemolytica was the major causative agent involved in calve pneumonic pasteurellosis in the study area. Although the infection rate varies, this finding is consistent with previous reports of [23]. Though Mannheimia haemolytica is a normal flora of the upper respiratory tract, suppressors of the host immune system favor the multiplication of Pasteurella species, leading to bronchopneumonia in purely pneumonic animals [36]. The reasons for increased susceptibility to $M$. haemolytica infection in stressed animals are primarily attributed to the breakdown of innate pulmonary immune barriers by stressors $[37,38]$. Although the percentage of isolation was relatively low $(28.8 \%)$ the possible role of $P$. multocida in the etiology and pathogenesis of calve pneumonia should not be under estimated [23].

Concerning the isolation rate of Pasteurella species in relation to the health status, it was higher in animals with respiratory illness (93.6\%) than no respiratory illness (54.1\%), among which Mannheimia haemolytica and Pasteurella multocida were isolated from $57.4 \%$ and $36.2 \%$ of calves with respiratory disease symptoms, while they were isolated from $32.4 \%$ and $21.6 \%$ of calves with no respiratory illness, respectively. In this study significant variation was observed in prevalence of Pasteurella species in calves with and without respiratory illness. This suggests the possible involvement of these bacteria in the genesis of pneumonia. 


\section{Conclusion}

This present finding revealed that pasteurellosis is one of the major diseases of calves in the study area in which M. haemolytica and P. multocida were found to be commonly involved in respiratory infections. Age, breed, sex, and livelihood were the risk factors associated with occurrence of respiratory infection. The study focuses on $M$. haemolytica and $P$. multocida. Therefore, further epidemiological investigation is needed to rule out the role of concurrent microbial causes of calf pneumonia, so as to design appropriate control and preventive measures at farm as well as national level.

\section{Abbreviations}

BAL: brocho alveolar lavage; BCS: body condition scores; BRD: bovine respiratory disease; Cl: confidence interval; DNS: deep nasopharyngeal swabs; LG: logistic regression; MCF: methods of colostrum feeding; TTA: transtracheal aspiration

\section{Declarations}

\section{Ethics approval and consent to participate}

The investigators treated animals with kindness and took proper care by minimizing discomfort, distress or pain. Basically, the study was conducted by taking swab samples from the nasal cavity of animals. In addition, there were no human subjects and no private data was taken except history related with management of animals. Nevertheless, Verbal consent was obtained from calves owners for inclusion of their calves in the study. Confidentiality of the data obtained was strictly followed in the study periods as well as they were told that the data is not to be used for any other purpose that was not intention of the study. Finally, ethical clearance was obtained from Haramaya University ethical review board.

\section{Consent for publication}

Not applicable

\section{Availability of data and materials}

The datasets used and/or analyzed during the study are available from the corresponding author on reasonable request.

\section{Competing interests}

The authors declare that none of them have financial or personal relationships with individuals or organizations that may have inappropriately influenced them in writing this paper and, therefore, declare that there is no competing interest.

\section{Funding}


No funding was obtained for this study

\section{Author contributions}

TM participated in the study design, field work and conducted the work. PW, AA and TF designed the study; provision test material, analyzed the statistics, performed data management and interpretation, prepared and wrote the manuscript. All authors read, evaluated and approved the final manuscript.

\section{Acknowledgments}

The researchers would like to acknowledge University of Gondor for the provision of valuable resource and laboratory facility and technical supports during the study period. We would also like to acknowledge farm owners who are highly appreciated for their tremendous cooperation and allowing us to take samples.

\section{Author details}

${ }^{1}$ Field Veterinarian, Amhara Livestock and Fishery Development Office of Sede woreda, East Gojam, Ethiopia. ${ }^{2}$ College of Veterinary Medicine, Haramaya University, P.O. Box 138, Dire Dawa, Ethiopia. ${ }^{3}$ College of Veterinary Medicine and Animal Sciences, University of Gondor, P.O. Box 196, Gondor, Ethiopia.

\section{References}

1. Kirchhoff J, Sabine U, Katherina G, Günther MK, Georg H. Three viruses of the bovine respiratory disease complex apply different strategies to initiate infection. J Vet Res. 2014;45:201-12.

2. Härtel H, Nikunen S, Neuvonen E, Tanskanen R, Kivelä SL, Aho P, Soveri T, Saloniemi H. Viral and bacterial pathogens in bovine respiratory disease in Finland. Acta Vet Scand. 2004;45(4):193-200.

3. Taylor JD, Fulton RW, Lehenbauer TW, Step DL, Confer AW. The epidemiology of bovine respiratory disease: What is the evidence for predisposing factors? The Canadian Veterinary Journal. 2010 Oct;51(10):1095.

4. Radostits OM, Gay CC, Blood DC, Hinchcliff KW. Veterinary Medicine. A Textbook of Disease of Cattle, Sheep, Pigs, Goats and Horses. 9th ed. London: WB. Saunders Ltd., United Kingdom.; 2000. pp. $1550-600$.

5. Lopez A. Respiratory system, thoracic cavity and pleura. In: Thomson's Special Veterinary Pathology, 3rd ed. 2001:125-95.

6. Kabeta T, Fikadu T, Zenebe T, Kebede G. Review on the pneumonic pasteurellosis of cattle. Academic Journal of Animal Diseases. 2015;4(3):177-84.

7. Campbell J. Enzootic Pneumonia of Calves and Shipping Fever Pneumonia. Veterinary Manual: MSD; 2012. 
8. Catry B. Pasteurella and Mannheimia species from calves: differentiation and antimicrobial resistance (Doctoral dissertation, Ghent University), 2005.

9. Kehrenberg C, Schulze-Tanzil G, Martel JL, Chaslus-Dancla E, Schwarz S. Antimicrobial resistance in Pasteurella and Mannheimia: epidemiology and genetic basis. Veterinary research. 2001 May 1; 32(3-4):323-39.

10. Adamu JY, Ameh JA. Pasteurellosis to mannheimiosis: Taxanomic changes. Nigerian Veterinary Journal. 2007;28(1):75-9.

11. Asaye $M$, Biyazen $H$, Bezie $M$. Isolation and characterization of respiratory tract bacterial species from domestic animals with pneumonic lungs from Elphora abattoir. Ethiopia Inter J Microbiological Res. 2015;6(1):13-9.

12. Hanzlicek GH, White BJ, Mosier D, Renter DG, Anderson DE. Serial evaluation of physiologic, pathological and behavioral changes related to disease progression of experimentally induced Mannheimia haemolytica pneumonia in post weaned calves. Am J Vet Res. 2010;71(3):359-69.

13. Pardon B, De Bleecker K, Dewulf J, Callens J, Boyen F, Catry B, Deprez P. Prevalence of respiratory pathogens in diseased, non-vaccinated, routinely medicated veal calves. Vet Rec. 2011;169:278.

14. Timsit E, Christensen H, Bareille N. Transmission dynamics of Mannheimia haemolytica in newlyreceived beef bulls at fattening operations. Vet Microbiol. 2013; 295-304.

15. Thomas A, Dizier I, Trolin A. Comparison of sampling procedures for isolating pulmonary mycoplasmas in cattle. Vet Res Commu. 2002;26:333-9.

16. Godinho KS, Sarasola P, Renoult E, Tilt N, Keane S, Windsor GD, Rowan TG, Sunderland SJ. Use of deep nasopharyngeal swabs as a predictive diagnostic method for natural respiratory infections in calves. Vet Rec. 2007;160:22-5.

17. Admasu MT, Hassen DJ. Major management and health problems of calves in smallholder dairy farms in selected areas of Dugda Bora, Arsi Negelle, Shashemene and Kofelle Woredas. Journal of Veterinary Science Technology. 2016;7(4):351.

18. Minda A, Abdisa B. Major Calf Health Problems and Exposing Risk Factors at Holeta Agricultural Research Center Dairy Farm, Holeta, Ethiopia. Global Veterinaria. 2016;17(1):05-14.

19. Mohamed RA, Abdelsalam EB. A review on pneumonic pasteurellosis (respiratory mannheimiosis) with emphasis on pathogenesis, virulence mechanisms and predisposing factors. Bulgarian Journal of Veterinary Medicine. 2008;11(3):139-60.

20. Carter GR. Diagnostic procedures in veterinary bacteriology and mycology. C. Charles and Thomas, publisher. 4th ed. USA. 1984. p. 3-19.

21. Quinn PJ, Morkey BK, Carter ME, Donnelly WJ, Lenard FC, Maguire D. Pasteurella species and Mannheimia haemolytica. Veterinary microbiology and microbial diseases, 1 st ed. London: Black Well Science Ltd. 2002:pp. 137-42.

22. Mac Faddin JF. Biochemical Tests for Identification of Medical Bacteria. The Williams and Wilkins Co, 2nd ed. 1999. 
23. Abera D, Sisay T, Birhanu T. Isolation and identification of Mannhemia and Pasturella species from pneumonic and apparently healthy cattle and their antibiogram susceptibility pattern in Bedelle District, Western Ethiopia. African Journal of Bacteriology Research. 2014 Dec 31; 6(5):32-41.

24. Muktar M, Fikadu G, Wondimu A, Bayu Y. Isolation of Pasteurella multocida and Mannheimia haemolytica from pneumonic calves and their antibiotic susceptibility in Harar and Haramaya, Eastern Ethiopia. Global Veterinaria. 2017;19(5):627-32.

25. Mohammed R. Bacteriological and histological examination of Pneumonic lungs of small ruminants slaughtered at Hashim Nure export abattoir. DVM thesis, AAU, FVM. Debre Zeit, Ethiopia. 1999:1-21.

26. Eshetu M. Pneumonic Pasteurellosis in sheep slaughtered at Addis Ababa abattoir. DVM thesis, FVM, Debre-Zeit, Addis Ababa University, Ethiopia. 1991.

27. Nurhusein Y. Aerobic bacterial Flora in the respiratory tract of apparently healthy sheep slaughtered in Dessie Abattror, North Eastern Ethiopia. Debre-Zeit: DVM thesis, FVM, Addis Ababa University, Ethiopia. 2005.

28. ASMARE AA, KIROS WA. Dairy calf morbidity and mortality and associated risk factors in Sodo town and its suburbs, Wolaita zone, Ethiopia. Slovak Journal of Animal Science. 2016 Mar 31; 49(1):4456 .

29. Konjit M, Endale B, Daniel H. Major management and health problems of calves in dairy farms in and around Mekelle. Ethiopia Rev elect Vet. 2013;14(2):1-13.

30. Mellado M, Lopez E, Veliz FG, De Santiago MA, Macias-Cruz U, Avendaño-Reyes L, Garcia JE. Factors associated with neonatal dairy calf mortality in a hot-arid environment. Livestock Sci. 2014;159:149-55.

31. Heinrichs AJ, Kehoe SI, Gehman AM, Jones CM, Tricarico JM. Case Study: Amylase Effects on Neonatal Rumen Development in Neonatal Dairy Calves. Profess Animal Sci. 2007;23(1):64-9.

32. Windeyer MC, Leslie KE, Godden SM, Hodgins DC, Lissemore KD, LeBlanc SJ. Factors associated with morbidity, mortality, and growth of dairy heifer calves up to 3 months of age. Preve Vet Med. 2014;113(2):231-40.

33. Yohannes A. Background Study on the pneumonic pasteurollosis in Ethiopia. Epidemiology, veterinary services, Ministry Of Agriculture. Addis Ababa, Ethiopia. 2001.

34. Radostits OM, Gay CC, Hinchcliff KW, Constable PD. Veterinary Medicine: A text book of the disease of cattle, horses, sheep, pigs and goats. 10th ed. London: Elsevier; 2007. pp. 674-762.

35. Chowdhury SA, Bhuiyan MSA, Faruk S. Rearing Black Bengal goat under semi-intensive management 1. Physiological and reproductive performances. Asia J Animal Sci. 2002;15:477-84.

36. Aiello SE, Mays A. The Merck Veterinary Manual. Whitehouse Station: Merck \& Co. Inc.; 1998. pp. 2032-3.

37. Martin WB. Respiratory infections of sheep. Comp Immunol Microbiol Infect Dis. 1996;19(3):171-79. 38. Pasteurellosis transmission risks between domestic and wild sheep 
Miller MW, Knowles DP, Bulgin MS, Clay BR, Cook WE, Srikumaran S, Scarfe AD, Layfield D. Pasteurellosis transmission risks between domestic and wild sheep, 2008.

\section{Figures}

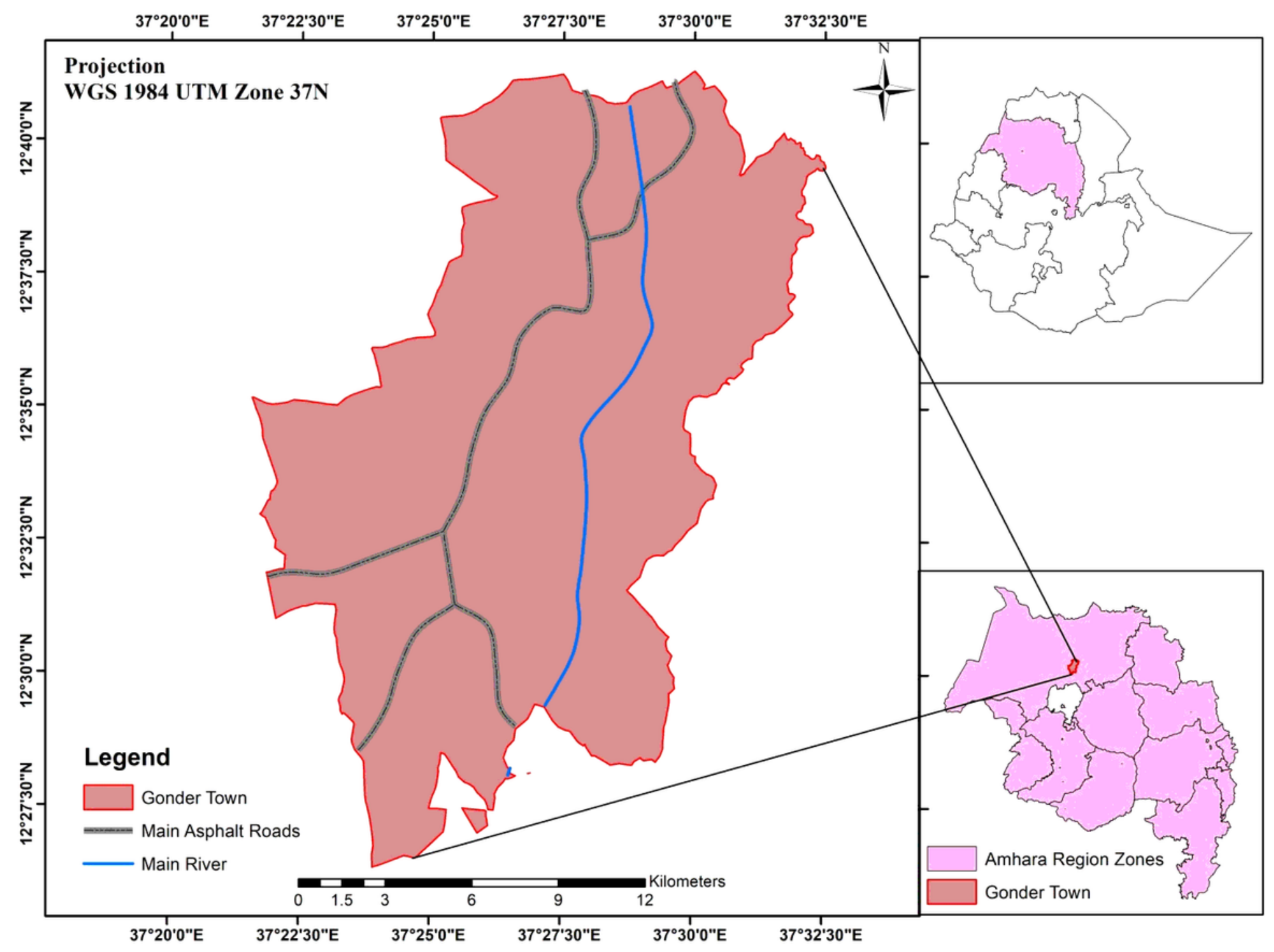

Figure 1

Map showing the Study area (Q-GIS version 3.10, http://osgeo4w-oslandia.com) 